$\xi=-1$

\title{
Reclamation of chemical wastes for the production of efficient concrete modifiers
}

\author{
Kosukhin M.M. ${ }^{1 *}$, Starostina I.V. ${ }^{1}$, Kosukhin A.M. ${ }^{1}$ \\ ${ }^{I}$ FSBEI HPE «Belgorod State Technological University named after V.G. Shukhov», \\ Belgorod, Russia (308012, Belgorod, Kostyukov str. 46) \\ *Corresponding author E-mail: mkosuhin@mail.ru
}

\begin{abstract}
The research concerning the obtaining of highly-efficient polyfunctional superplasticizers for concretes on the basis of chemical production waste with the purpose of their reclamation and improving the environmental situation in the populated areas has been carried out. The highly-efficient concrete admixtures on the basis of various wastes have been synthesized and patented. A new efficient modifier based on pyrocatechin production waste has been suggested. The colloid and chemical properties of the obtained modifier and its influence on physical, mechanical and maintenance-engineering characteristics of concrete have been researched. Its comparative evaluation with the known plasticizing admixtures has been presented. It has been demonstrated that the superplasticizer under study is the most efficient in terms of plasticizing activity and improving the physical and mechanical properties of concretes and concrete mixes and has a polyfunctional effect. Its application allows increasing the concrete mixes flowability and concrete strength, reducing the concrete consumption, providing the concrete with fungicidal properties, cutting the production costs and solving ecological problems of the chemical production waste recycling.
\end{abstract}

Keywords: Chemical industry waste, environmental safety, plasticizing activity, pyrocatechin production waste, waste resin

\section{Introduction}

Since the middle of the last century, along with the broad-scale development of housing construction, the building of industrial enterprises has been developing rapidly as well. Many of these enterprises are city-forming, and by now they have found themselves deeply inside populated areas or next to them. The overwhelming majority of industrial enterprises generate a wide range of waste products, having an adverse impact on the environment. In this regard the problem of recycling waste, especially of chemical production, is highly topical.

The problem of production waste recycling has attracted the attention of the President of the Russian Federation. Over the years the amount of accumulated waste in Russia has reached $90 \mathrm{bln}$. tons and continues to increase by $3.5 \mathrm{bln}$. tons each year. Over $75 \%$ of these wastes is not treated or recovered, but only dumped at landfills, or worse, at illegal dumping sites. The problem of waste recycling becomes more and more urgent each year, and its impact on the living conditions and on the population health increases. The most hazardous are wastes, generated as a result of chemical industries operation, as they can react with soil and water. The chain of such reactions can be unpredictable; the consequences can be dangerous and irremovable. The unpredictability in the chemical processes of these wastes is due to their long decay period. The disposal of chemical industry production waste requires the special machinery and equipment. The delayed dealing with waste provokes the negative effects on the economy development; elimination of these problems (as the experience of the highly industrialized countries demonstrates) is possible only at the active state intervention.
In the Western world the problems of environmental damage were being solved, especially at early 70 -ies of the past century, by the direct budgetary funding. Neither in Europe, nor in America had the attempts to invite for these purposes the private sector investments any success. The disposal and utilization of waste is a technological and industrial problem, which must be solved by manufacturers in the first place, and the environmental experts should only control their solutions. It's interesting that the waste market, created in the Western countries, amounts nowadays to over 500 bln dollars. In Russia this problem mostly remains ecological, i.e loss-making. Solving the problem of recycling chemical substances and wastes is a key factor of ensuring the environmental safety of the country and its sustainable development. A sound option in this regard is the opportunity of their recovery.

\section{The methodology}

In the course of this work, the colloid and chemical properties of modified mineral suspensions, which are most widely used in building industry, have been studied.

The flowability of modified cement suspensions was researched by mini-cone method, according to the Gosstroy's Concrete and Reinforced Concrete Research Institute (CRCRI) methodology [1], which consists in determining the spread diameter of a cement suspension under the action of gravity.

The rheological properties of cement grouts and mortars were researched and the batching of cement concrete was carried out according to the methodology, developed in CRCRI, concerning the application of admixtures of various kinds in pre-cast and mass concrete technology [2]. 
To exclude the factor of the dispersion phase and dispersion medium instability at the interaction of cement with water when studying the influence of the polyfunctional modifier on colloid and chemical properties, the suspension of chalk was used as a standardized test suspension. $\mathrm{CaCO}_{3}$ has low solubility (solubility product $1 \cdot 10^{-8}$ ) and contains cations, which are part of most clinker minerals of cement. The polyfunctional modifier's mechanism of action was studied by carrying out the standard tests for colloid and chemical properties and modified mineral suspensions.

The rheological parameters of suspensions were researched by using a rotation viscometer «Reotest-2». The concentration of polyfunctional modifier $\left(\mathrm{C}_{\mathrm{m}}\right)$ was calculated in per cent for weight for dry matter from the amount of dispersion phase. In the course of the research the dependence between shear stress value and shearing rate was determined. On the basis of the obtained findings the rheological curves were plotted, by means of which the yield value $[\mathrm{tau}]_{0}$ and plastic viscosity [eta $]_{\mathrm{pl}}$ were found.

The aggregative stability of chalk suspensions was evaluated by the most probable particle radius, by means of sedimentation analysis.

To find the electrokinetic properties of the surface the flow potential method was used with account of the surface conductivity, which allows determining [zeta]-potential in concentrated suspensions.

To determine the composition and structure of oligomeric molecules the liquid and gas-liquid chromatography, ultraviolet and infrared spectroscopy, NMR-spectroscopy, conductometry and potentiometry were used. The molecular weight of the synthesized oligomers was determined by means of cryoscopy.

The modifiers' mechanism of action was determined by means of physical and chemical research methods. For this purpose the water solutions of modifiers, the hardening kinetics of cement paste, the alteration of phase composition of cement stone samples with additives, the phase transformations in models systems and clinker minerals at hydration and hardening were studied.

\section{The main part}

The chemical industry wastes, after the preliminary treatment, can be successfully applied as the low-cost raw stuff for producing various chemical admixtures for concretes [3, 4]. The development of technology and practical application of the up-to-date concrete is based, in the first place, on using various types of additives - modifiers, the application of which is one of the most universal, available and flexible means of controlling the concrete technology and regulating its properties. The plasticizers and superplasticizers $[5,6]$ are of special interest in this sphere. Added at small amounts $(0,1 \ldots 2 \%$ of the cement weight), they allow regulating the concrete mixes properties and obtaining concretes with the predictable properties for the specified operating conditions. There are many classes of various concrete superplasticizers $[7,8]$ known; their properties, mechanism of action and spheres of application have been thoroughly studied. But at the same time, they are normally the specially synthesized chemical substances, expensive and requiring the specialized chemical technologies and equipment for their production. So, the search of new, highlyefficient and low-cost superplasticizers has always been a relevant task. At the same time, these chemical substances are contained in various amounts in waste products and byproducts, generated at their production. The results of the preliminary studies have demonstrated that most of these wastes can be successfully used in concrete admixtures production, cutting their production costs considerably, while preserving the quality.

Since the 80-ies of the past century, the authoring team of BSTU named after V.G. Shukhov has been carrying out works in the sphere of synthesizing the highly-efficient admixtures for concretes and building mortars on the basis of various chemical industries wastes, as the cheapest and the most available raw stuff. An important aspect at this is also solving the ecological problem of the production waste recycling.
As a result of multi-year research the following superplasticizers have been synthesized and patented: SBR, SB-3, SB-2A, SB-5, SB-7 and others [9-12], the application of which allows not only cutting the concrete production costs, but also solving ecological problems concerning the hazardous chemical industries waste recycling. In Tab.1 the utilized chemical industry wastes and the stages of production technology of some synthetic additives, having the highest plasticizing activity, are presented. Due to the fact that the plasticizing effect of a superplasticizer is determined by the oligomeric chains in its composition, a hypothesis has been suggested that as a result of distillation, high-temperature treatment, condensation and polymerization processes the still residues of pyrocatechin production contain oligomers with phenolic oxygroups. This gives preconditions of using them as efficient superplasticizers for concretes. The testing of synthesizing a polyfunctional modifier on the basis of pyrocatechin production waste (SB-4), its properties and its influence on the properties of concretes and concrete mixes has been performed.

Table 1: The efficient superplasticizers for mineral dispersions based on resorcine production waste

\begin{tabular}{|c|c|c|}
\hline \multicolumn{2}{|r|}{ Plasticizers } & Monomer \\
\hline Name & Production technology & Production waste, (\% of weight) \\
\hline SB-3 & $\begin{array}{l}\text { 1. Polycondensation } \\
\text { with formaldehyde } \\
\text { 2. Neutralization }\end{array}$ & $\begin{array}{l}\text { Resorcine (monomeric phenols }(20- \\
40) \text {, dimeric phenols }(20-35) \text {, trimeric } \\
\text { phenols }(10-25) \text {, resorcine }(2-20))\end{array}$ \\
\hline SB-5 & $\begin{array}{l}\text { 1. Polycondensation } \\
\text { with furfurol } \\
\text { 2. Neutralization }\end{array}$ & $\begin{array}{l}\text { Resorcine (monomeric phenols }(20- \\
40) \text {, dimeric phenols }(20-35) \text {, trimeric } \\
\text { phenols }(10-25) \text {, resorcine }(2-20))\end{array}$ \\
\hline
\end{tabular}

The resin waste of pyrocatechin production is a still residue - the viscous liquid substance from dark green to black in color, setting at temperature below $10-12^{\circ} \mathrm{C}$, with density $1.25 \mathrm{~g} / \mathrm{cm}^{3}$. It contains: pyrocatechin $-15-16 \%$, resorcine $-2-4 \%, \mathrm{NaCl}-$ up to $20 \%, \mathrm{Na}_{2} \mathrm{SO}_{4}-10-12 \%$ and tar products. The method of obtaining superplasticizer is simple; it can be obtained right at concrete mix plants by the following technology: in a tank, equipped with a forced action mixer, the estimated amount of water is poured (70\% of the final product weight), in which $5 \%$ of $\mathrm{NaOH}$ or $\mathrm{KOH}$ is dissolved. Then the estimated amount of the resin waste (25$26 \%$ ) is added to the prepared alkaline solution and is mixed until completely dissolved. The obtained ready-to-use water solution is fed through the batcher with the gauged water into the concretemixing machine. The superplasticizer is a low-hazardous substance and according to GOST 12.1.007.76 belongs to hazard class IV.

The conditions of pyrocatechin synthesis predetermine the formation of a production waste with unique properties, in terms of using it as a polyfunctional concrete modifier. The presence of sodium chloride and sulfate allows including it into the category of accelerating and antifreezing agents for concretes. Pyrocatechin and resorcine, contained in the waste, are fungicidal by their nature, which provides the superplasticizer with fungicidal properties. So, the application of this superplasticizer allows, along with regulating the rheological properties, altering purposefully at the same time a number of basic properties of concretes and concrete mixes.

The colloid and chemical properties of the superplasticizer under study and its influence on physical and mechnanical properties of concretes and concrete mixes have been studied. The comparative analysis with the well-known domestic equivalent - fluidifier C-3 - has been performed. The research was carried out on the cement of the Belgorod cement plant CEM I 42,5H.

To study the mechanism of the SB-4 superplasticizer's plasticizing effect, its influence on rheological properties of cement paste was tested by means of a rotation viscometer with coaxial cylinders «Reotest-2.1».

The research of cement paste rheological parameters in the presence of the superplasticizer has shown that they are typical viscoplastic substances. Their flow is the most accurately described 
with the Ostwald equation: $\tau=k \cdot \dot{\gamma}^{n}$. In the range of average dosages of SB-4 the linear part of rheological curves increases considerably, and the suspension flow is described with the Bingham equation: $\tau=\tau_{0}+\eta_{\text {пл }} \dot{\gamma}$. With the further increase of dosages the flow from thixotropic becomes Newtonian, and the rheological curves are described with the Newtonian equation: $\tau=\eta_{\text {пл }} \dot{\gamma}$

By these rheological curves the yield point $\tau_{0}$ and the plastic viscosity [eta $]_{\mathrm{pl}}$ were determined, the dependence of which on SB-4 and S-3 dosage are presented in Fig. 1.

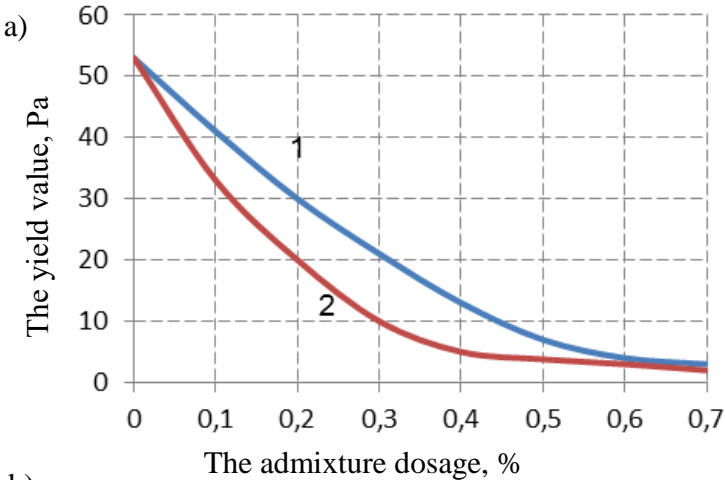

b)

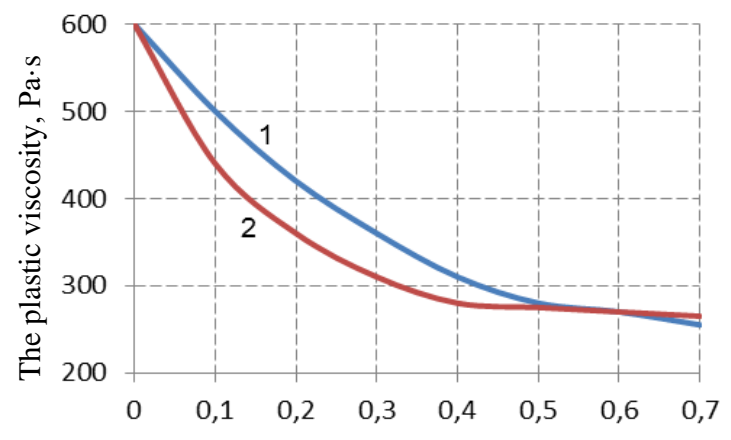

The admixture dosage, $\%$

Fig. 1: The dependence of yield value (a) and plastic viscosity (b) of cement paste on the superplasticizers dosage: 1 - SB-4;2-S-3.

At the increase of SB-4 and S-3 concentration the yield value at first drops sharply, then its decrease is slowed down, and at the optimal dosage it tends to zero. The plastic viscosity also drops sharply at first, but then reaches a certain minimum value.

The reduction of $[\mathrm{tau}]_{0}$ virtually to zero at optimal admixture dosages is conditioned with the decrease of individual contact strength to values, comparable with thermal motion energy. The decrease of the plastic viscosity is first of all due to the release of the immobilized water and the increase, as a result, of the dispersion medium abundance. Increase of the water layers thickness between particles causes the reduction of the friction between moving layers and the decrease of plastic viscosity.

The aggregative stability of cement suspensions was evaluated by the most probable radius of particles, formed in cement-water system by means of sedimentation analysis. The differential curves of radial distribution of cement particles at various SB-4 and S-3 superplasticizers dosages are presented in Fig. 2.

The obtained data demonstrate that increasing the SB-4 dosage results in the more narrow distribution of particles and the shifting of the distribution maximum to the smaller radius values. The dependence of the most probable radius of cement particles on the superplasticizers dosages are presented in Fig. 3.

To exclude the influence of cement and water interaction, causing the change of dispersion phase and dispersion medium composition, and for the more complete colloid and chemical research of the superplasticizer's influence on adsorption and electrokinetic potential, a model system - the chalk suspension - was used.

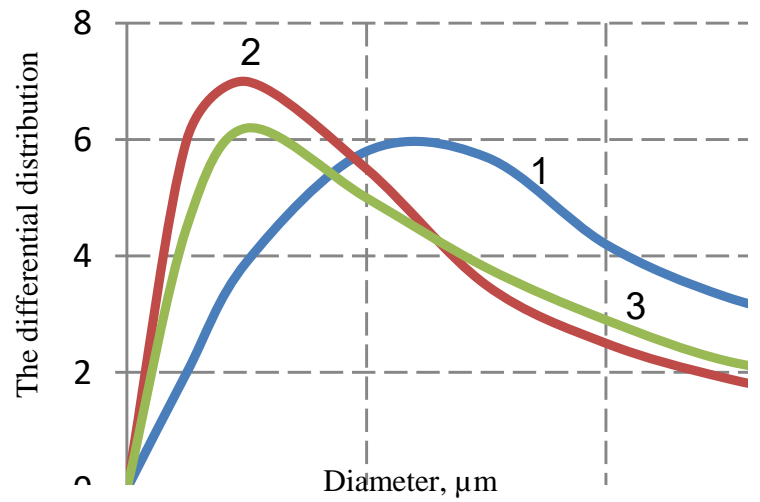

Fig. 2: The differential curves of cement particles distribution for CEM I 42,5H: 1 - no admixture; 2 - SB-4; 3 - S-3

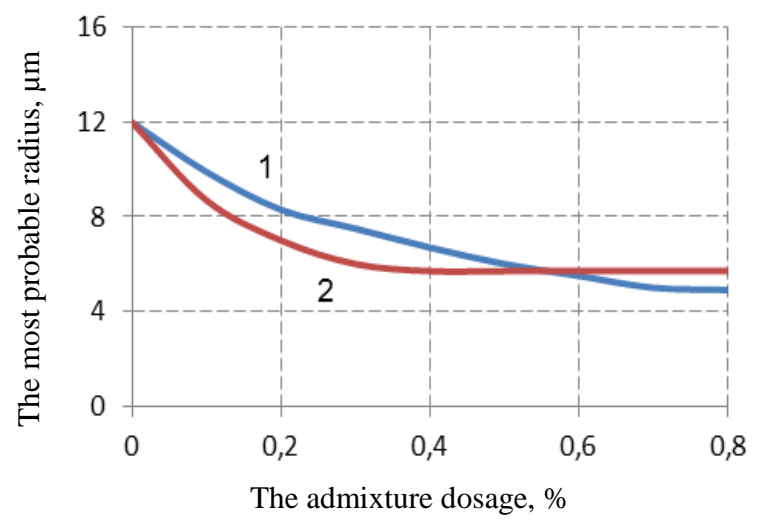

Fig. 3: The influence of admixture dosage on the most probable radius of particles for cement CEM I 42,5H: 1 - SB-4; 2 - S-3.

The alteration of rheological parameters and aggregative stability of mineral suspensions is conditioned by the modifying of the dispersion phase surface. The study of the adsorption isotherms has shown that the adsorption of admixtures on chalk is of monomolecular character.

The study of electrokinetic potential [zeta] of chalk particles, measured by means of the flow potential method at various concentrations, has demonstrated that the surface of the unmodified chalk has a slightly excess negative charge, which is indicated by the slight negative value of the [zeta]-potential. The increase of superplasticizer dosage results in the alteration of [zeta]-potential absolute values in the negative range from -5 to $-40 \mathrm{mV}$, which is explained by the presence of anion-active groups in SB-4, the oligomeric molecules of which are adsorbed on the surface of the dispersion phase.

When comparing the adsorption data, the electrokinetic potential values and rheological properties of chalk suspension, it should be mentioned that the concentration of admixtures, at which the monolayer formation on the surface of chalk particles is completed and the $\xi$-potential reaches its maximum, corresponds to yield value amounting to zero.

Judging from the obtained data, the improvement of concretes and concrete mixes efficiency consists in the plasticizing effect of the superplasticizer under study. The admixtures molecules are adsorbed on the surface of particles, forming a monomolecular layer. The admixtures are adsorbed on the particles surface due to the dispersion forces of interaction between the admixture's aromatic rings system and the particles surface. As the admixtures are anion-active substances, the charge of the particles surface becomes more negative, which results in the increase of the particles' repulsion forces. The formation of hydrate layers over the particles due to the presence of hydrophilic groups in the superplasticizer molecules also contribute to this process. As a result, the repulsion forces begin to exceed molecular attraction forces, which reduce the coagulation contact energy to the values, comparable with 
thermal motion energy. The thixotropy, conditioned by the particles interaction, virtually disappears. The yield value drops to zero, the plastic viscosity is reduced considerably. The aggregates are peptized and the aggregative stability of suspensions is increased.

An essential characteristic of the concrete durability is its frost resistance, which is determined with its pore structure. Measuring the limiting wetting angles of the surface has demonstrated that the adsorption of SB-4 results in the decrease of the surface tension at the solid-solution boundary by $20-25 \mathrm{~mJ} / \mathrm{m}^{2}$, which indicates the increase of the surface hydrophility. At this the air entrainment into the concrete mix is increased both at the constant W/C and at the equal flowability by 3-5\%; the volume of arbitrarily closed fine spherical pores is increased, while the opened capillary porosity volume is reduced. All this considerably improves the frost resistance of concretes [13].

The SB-4 superplasticizer increases the cement mix flowability from $2-4 \mathrm{~cm}$ to $20 \mathrm{~cm}$ and more, without the concrete strength loss, at the constant $\mathrm{W} / \mathrm{C}$ rate, reduces the water requirement of a concrete mix up to $20 \%$ for equally-flowable concrete mixes, and reduces the cement consumption up to $20 \%$. The peptizing effect of SB-4 results in the formation of more fine-crystalline structure, which contributes to the compaction of cement stone and reducing the amount of microcracks inside the concrete and on its surface. The hardening agents, contained in SB-4, accelerate the hydration processes and structure formation of concrete. The introduction of a hardening accelerating agent reduces the clinker particles charge, which in the initial period results in the thinning of the adsorbed water layer, preconditioning the possibility to obtain the more compact concrete. SB-4 quickens the interaction of clinker cement phases with water and, consequently, the rate of concrete hardening.

At service in biologically active media, the concretes are exposed to corrosion damage by mold fungi [14], which makes it necessary to protect them from biodeterioration. The presence of inorganic hardening accelerating substances and fungicides in the composition of SB-4 superplasticizer can be used for providing the concrete with fungicidal properties.

As follows from the research (tab. 2), adding the SB-4 polyfunctional modifier to concrete's composition in amount of $0.7 \%$ of cement weight completely inhibits the mold fungi growth, taken from the surface of the similar unprotected concrete samples.

Table 2: The properties of cement paste and cement stone with S-3 and SB-4 superplasticizers

\begin{tabular}{|c|c|c|c|c|c|c|c|c|c|}
\hline \\
\hline \multirow[b]{2}{*}{ № } & \multirow[b]{2}{*}{$\begin{array}{l}\text { Type of } \\
\text { admix- } \\
\text { ture }\end{array}$} & \multirow{2}{*}{$\begin{array}{c}\text { Amou } \\
\text { nt of } \\
\text { ad- } \\
\text { mix- } \\
\text { ture } \\
\%\end{array}$} & \multirow[b]{2}{*}{$\mathrm{W} / \mathrm{C}$} & \multirow{2}{*}{$\begin{array}{c}\text { Cement } \\
\text { paste } \\
\text { sprea- } \\
\text { da- } \\
\text { bility, } \\
\text { mm }\end{array}$} & \multirow{2}{*}{$\begin{array}{c}\text { Average } \\
\text { density } \\
\text { after } 28 \\
\text { days, } \\
\mathrm{g} / \mathrm{cm}^{3}\end{array}$} & \multicolumn{2}{|c|}{\begin{tabular}{|c} 
Compression \\
strength $\mathrm{R}_{\text {сж, }}$, \\
$\mathrm{MPa}$ \\
\end{tabular}} & \multirow{2}{*}{$\begin{array}{c}\text { Cement } \\
\text { stone } \\
\text { porosity, } \\
\%\end{array}$} & \multirow{2}{*}{$\begin{array}{c}\text { Water } \\
\text { adsorp- } \\
\text { tion, } \\
\%\end{array}$} \\
\hline & & & & & & $\begin{array}{l}\text { with- } \\
\text { out } \\
\text { fungi }\end{array}$ & $\begin{array}{c}\text { contam- } \\
\text { inated } \\
\text { with } \\
\text { fungi } \\
\text { spores }\end{array}$ & & \\
\hline 1 & $n / a$ & & 0.3 & 55 & 2.200 & 54.0 & 52.2 & 25.0 & 15.1 \\
\hline 2 & S-3 & 0.45 & 0.3 & 80 & & 54 & & & 14.9 \\
\hline 3 & S-3 & 0.45 & 0.26 & 60 & 2.215 & 59.3 & 58.5 & 24.8 & 14.7 \\
\hline 4 & SB-4 & 0.7 & 0.3 & 160 & 2.225 & 54.4 & 54.4 & 25.9 & 14.0 \\
\hline 5 & SB-4 & 0.4 & 0.26 & 110 & 2.222 & 60.1 & 61.1 & 26.1 & 14.3 \\
\hline
\end{tabular}

The strength characteristics of cement stone with SB-4 are not reduced at the contamination with mold fungi spores; on the contrary, they slightly increase due to the increase of cement paste flowability, reduction of W/C ratio, reduction of the microcracks amount in concrete and compaction of its structure. Besides, the presence of pyrocatechin and resorcine in SB-4, which are fungicides, also inhibits the mold fungi growth.

\section{Conclusion}

So, the obtained experimental data confirm that the superplasticizer under study is the most efficient in terms of plasticizing activity and improving physical and mechanical properties of concretes and concrete mixes and has polyfunctional effect. Its application allows increasing the concrete mixes flowability and concrete strength, reducing the cement consumption, providing the concrete with fungicidal properties, cutting the production costs and solving ecological problems of the chemical production waste recycling.

\section{Acknowledgement}

The article has been prepared within the framework of the Flagship University Development Program on the basis of BSTU named after V.G. Shukhov.

\section{References}

[1] Recommendations on control of physical and chemical composition and quality of S-3 superplasticizer, Moscow: CRCRI, (1984).

[2] Methodological recommendations on evaluating the additives efficiency, Moscow: CRCRI, (1979), p.24.

[3] Kosukhin M.M., Poluektova V.A., "Solving the ecological problems of populated areas by the reclamation of chemical industry waste for concrete superplasticizers production", The collected works of the 2nd International research and application conference "Energy saving and ecology in housing and utilities infrastructure and urban development", (2013), pp. 160-164.

[4] Kosukhin M.M., Shapovalov N.A., Denisova Yu.V., "Vibropressed concretes with various types of plasticizing additives", Proceedings of higher educational institutions. Construction, No. 6, (2007), pp. 26-29.

[5] Kosukhin M.M. Shapovalov N.A. Kosukhin A.M., "Colloidchemical bases on creation of multifunctional modifiers of concrete mix and concrete", Solid State Phenomena, Vol. 265, (2017), pp. 331-336.

[6] Poluektova V.A., Shapovalov N.A., Kosukhin M.M., Slyusar A.A., "Plasticizing additives for water mineral dispersions on the basis of oxyphenol oligomers", Advances in Natural and Applied Sciences, Vol. 8, No.5, (2014), pp.373-379.

[7] Kosukhin M.M., Regulating the properties of concretes and concrete mixes with complex additives with various hydrophilic groups, Belgorod: BSTU publishing office, (2005), p. 194

[8] Kosukhin M.M., Shapovalov N.A., "The theoretical aspects of superplasticizers mechanism of action", Concrete and reinforced concrete, No.3, (2006), pp. 25-27.

[9] Lomachenko V.A., Kosukhin M.M., Lomachenko S.M., Shablitsky V.N., "The action of SB-3 superplasticizer on concretes and concrete mixes", Building materials, No.6, (2005), pp. 64-65.

[10] Shapovalov N.A., Slyusar A.A., Kosukhin M.M., Mukhachev O.V., "SB-5 superplasticizer as a modifier for obtaining binding mixtures with low water demand and concretes based on them", Concrete and reinforced concrete, No.6, (2001), pp. 2-4.

[11] Babin A.A., Kosukhin A.M., Kosukhin M.M., Shapovalov N.A., "Superplasticizer for concretes based on light pyrolysis resin", Building materials, No.7, (2008), p. 44.

[12] Kosukhin M.M., Kosukhin A.M., Shapovalov N.A., "Composite binder for highly frost-resistant road concretes", Bulletin of Belgorod State Technological University named after V.G. Shukhov, No.1, (2010), pp. 51-53.

[13] Kosukhin M.M., Shapovalov N.A., "Improving the frost-resistance of expanded-clay concrete by using polyfunctional modifiers", Building materials, No.11, (2006), pp. 66-67.

[14] Kosukhin M.M., Ogrel L.Yu., Pavlenko V.I., Shapovalov I.V., "Bioresistant cement concretes with polyfunctional modifiers", Building materials, No. 11, (2003), pp. 48-49. 